\title{
News from China 201601
}

\section{The Ninth China Patent Week}

The 9th China Patent Week was inaugurated throughout the country on November 30,2015 , centering on the theme "boosting IP utilization and protection to aid mass entrepreneurship and innovation". During the week, the national intellectual property system intensively pushed the policies and service information of intellectual property via online and offline ways. Meanwhile, several services were launched among enterprises, colleges and academic institutes.

This patent week took "simple form, rich content, strengthening services, highlighting effectiveness" as the principle. The China Intellectual Property Network was as the main online venue, and here were no offline venue and opening ceremony. With the in-depth implementation of innovation driving development strategy, national intellectual property strategy, and the theme of intellectual property services for " mass entrepreneurship and innovation", a number of key work were launched in the patent week, including: 1) promoting the optimization and coordination of IP public policy system and improving environment for mass entrepreneurship and innovation; 2) improving IP public service system and providing more platforms for mass entrepreneurship and innovation; 3) further propelling construction of IP financial system; 4) promoting construction of IP commercialization system and building an ecological innovation system; 5) improving the innovation capability of innovators and inspiring the innovation enthusiasm; 6) facilitating construction of IP culture and creating better environment for innovation.

By visiting the main online venue of this China Patent Week, that is, the China Intellectual Property Network, people could see the activities during the event throughout the country in real time. At the same time, the local intellectual property offices gathered intellectual property public resources actively. Targeted services were launched around five key aspects, including optimization of intellectual property policies, construction of public intellectual property service system, financial innovation of intellectual property, operation system of intellectual property, and subject ability enhancement of intellectual property to demonstrate work results intensively and promote related work continuously.

The first patent week was held in 2007. The annual China Patent Week has gradually grown influence and become a comprehensive platform for combining patent resources, facilitating patent use and fostering patent culture with the in-depth implementation of innovation driving development strategy and intellectual property strategy and solid progress of intellectual property power country in recent years.

http://www.sipo.gov.cn/ztzl/ndcs/zgzlznew/ http://www.sipo.gov.cn/zscqgz/2015/201511/t20151125_ 1207978.html

http://www.sipo.gov.cn/ztzl/ndcs/zgzlznew/xgbd/201512/ t20151202_1211962.html

Ying-Qi Xu

Chengdu Library of Chinese Academy of Sciences

\section{State intellectual property office completed the task of four countries' patent data comparison and complement}

In recent years, with the development of Chinese patent data exchange, State Intellectual Property Office (SIPO) has enlarged the range of foreign patent data constantly through data exchange. At present, SIPO has finished comparison and complement of American, Japanese, European, and Koran full-text patent data.

In order to ensure the integrity of patent data gotten from exchange and process existing foreign patent data to use for intraSIPO business system and public service, SIPO compared the patent data exchanged from America, Japan, Europe and Korea with authoritative documents and communicated with relative bureau about missing documents found from comparison to complete data on the basis of authoritative documents published by five bureaus' cooperation framework. Via several rounds of analysis, communication and data supplement, until now, the America, Japan, Europe and Korea full-text patent data obtained by SIPO have brought into correspondence with authoritative documents published by each country, which ensure the integrity of data applied for internal investigative system and public.

http://www.sipo.gov.cn/zscqgz/2015/201511/t20151113_ 1202723.html.

Ying-Qi Xu

Chengdu Library of Chinese Academy of Sciences

\section{China-Japan, China-Denmark, and China-Portugal PPH pilots have been extended}

Patent Prosecution Highway (PPH) is a quick application method for patent applicants who apply patents both in CN State Intellectual Property Office (SIPO) and abroad. When an applicant receives a final ruling from a first patent office that at least one claim is allowed, the applicant may request fast track examination of corresponding claim(s) in a corresponding patent application that is pending in a second patent office.

PPH allows both countries to benefit from work previously done by the other office, in turn reducing examination workload. It also allows applicants to obtain corresponding patents faster, more efficiently, and with improved quality, which benefits both the 
applicants and industrial circles. For example, under the China-Japan PPH pilot, the average processing time in China for Japanese corporations who have granted patents in Japan could decrease from 1 year to less than half year.

Currently, Patent Offices which have signed agreements with SIPO and launched PPH pilots includes: Japan Patent Office, United States Patent and Trademark Office, German Patent and Trade Mark Office, Korean Intellectual Property Office, Federal Service for Intellectual Property, Patents and Trademarks (Russian Federation), Danish Patent and Trademark Office, Finnish Patent and Registration Office, Mexican Institute of Industrial Property, Austrian Patent Office, polish patent office, Intellectual Property Office of Singapore, Canadian Intellectual Property Office, National Institute of Industrial Property (Portugal), Spanish Patent and Trademark Office, European Patent Office, Israel Patent Office, United Kingdom Intellectual Property Office, Icelandic Patent Office, and Swedish Patent and Registration Office.

CN SIPO has launched PPH pilots with Japan Patent Office, Danish Patent and Trademark Office, and National Institute of Industrial Property (Portugal) on November 1, 2011, January 1, 2013, and January 1, 2014 respectively. The terminal time of the China-Japan PPH pilot was October 31, 2011, and the terminal time of the China-Denmark, China-Portugal PPH pilot was December 31 , 2015. Recently, agreements have been signed between CN SIPO and these three patent offices, to extent their PPH pilots for three years on the next day of their terminal time, and remain the same framework, criteria, and principles.

http://www.sipo.gov.cn/ztzl/ywzt/pph/js/201311/t20131104_ 874613.html

http://www.sipo.gov.cn/ztzl/ywzt/pph/js/201311/t20131104_ 874615.html

http://www.ccin.com.cn/ccin/news/2011/10/18/203716.shtml

http://www.sipo.gov.cn/ztzl/ywzt/pph/zxdt/201512/t20151221_ 1220070.html

http://www.sipo.gov.cn/ztzl/ywzt/pph/zxdt/201512/t20151221_ 1220071.html

Ji-Qiang Shi

Chengdu Library of Chinese Academy of Sciences

\section{Ceremony for the 17th China Patent Awards was held in Beijing}

The ceremony for the 17th China Patent Awards which was hosted by State Intellectual Property Office (SIPO) and World Intellectual Property Office (WIPO) was held in Beijing on December 15, 2015.

China Patent Awards started from 1989, to 17 sessions have been successfully held since then. In this session, 20 China Patent Golden Awards such as "Preparation and properties of a Hydrogenation Conversion Catalyst for heavy oil and residual oil", 5 China Golden Design Awards such as "Artificial cochlea implant", 507 China Excellent Patent Awards such as "cloned attenuated mycoplasma hyopneumoniae", and 57 China Excellent Design Awards such as "bathtub (WHALE)"were awarded. Shenzhen Intellectual Property Office and 7 other Intellectual Property Offices were awarded as the Best Organization Awards. Hubei Intellectual Property Office and 19 other Intellectual Property Offices won the Excellent Organization Awards. Zhang-Li and 7 other academicians were selected as the Best Referees. Compared with the last session, China Excellent Patent Awards increased by $24 \%$.

For over 20 years, China Patent Awards have awarded a large number of excellent patents and have become the important marks of the development of China's independent innovation. Chinese innovation activities have become significantly active in recent years. The number of patent applications has ranked first for four consecutive years in the world, and the number of PCT international patent applications ranked third in the last year. To fully develop the guiding function of China Patent Awards, SIPO has introduced two prominent features. Firstly, the selection process becomes more scientific and mass participation is enhanced. Secondly, the new award system pays more attention to advanced technologies, and emphasizes the practical benefits and contribution to the society. Therefore, the guiding role of China Patent Awards becomes clearer, and the results become more credible.

http://www.sipo.gov.cn/zscqgz/2015/201512/t20151216_ 1218026.html

http://www.sipo.gov.cn/zscqgz/2015/201512/t20151204_ 1212868.html

http://english.sipo.gov.cn/news/official/201512/t20151223_ 1220938.html

Ji-Qiang Shi

Chengdu Library of Chinese Academy of Sciences 